\title{
Multi-part Left Atrium Modeling and Segmentation in C-Arm CT Volumes for Atrial Fibrillation Ablation
}

\author{
Yefeng Zheng ${ }^{1}$, Tianzhou Wang ${ }^{1}$, Matthias John ${ }^{2}$, \\ S. Kevin Zhou ${ }^{1}$, Jan Boese ${ }^{2}$, and Dorin Comaniciu ${ }^{1}$ \\ 1 Image Analytics \& Informatics, Siemens Corporate Research, Princeton, NJ, USA \\ 2 Healthcare Sector, Siemens AG, Forchheim, Germany \\ yefeng.zheng@siemens. com
}

\begin{abstract}
As a minimally invasive surgery to treat left atrial (LA) fibrillation, catheter based ablation uses high radio-frequency energy to eliminate potential sources of the abnormal electrical events, especially around the ostia of pulmonary veins (PV). Due to large structural variations of the PV drainage pattern, a personalized LA model is helpful to translate a generic ablation strategy to a specific patient's anatomy. Overlaying the LA model onto $2 \mathrm{D}$ fluoroscopic images provides valuable visual guidance during surgery. A holistic shape model is not accurate enough to represent the whole shape population of the LA. In this paper, we propose a part based LA model (including the chamber, appendage, and four major PVs) and each part is a much simpler anatomical structure compared to the holistic one. Our approach works on un-gated C-arm CT, where thin boundaries between the LA blood pool and surrounding tissues are often blurred due to the cardiac motion artifacts (which presents a big challenge compared to the highly contrasted gated CT/MRI). To avoid segmentation leakage, the shape prior is exploited in a model based approach to segment the LA parts. However, independent detection of each part is not optimal and its robustness needs further improvement (especially for the appendage and PVs). We propose to enforce a statistical shape constraint during the estimation of pose parameters (position, orientation, and size) of different parts. Our approach is computationally efficient, taking about $1.5 \mathrm{~s}$ to process a volume with $256 \times 256 \times 250$ voxels. Experiments on $469 \mathrm{C}$-arm CT datasets demonstrate its robustness.
\end{abstract}

\section{Introduction}

About $15 \%$ of all strokes (which is the third leading cause of death in the USA) are caused by atrial fibrillation (AF) [1]. As a widely used minimally invasive surgery to treat $\mathrm{AF}$, the catheter based ablation procedure uses high radiofrequency energy to eliminate the sources of ectopic foci, especially around the ostia of the appendage and the pulmonary veins (PV). Automatic segmentation of the left atrium (LA) is important for the pre-operative assessment to identify

G. Fichtinger, A. Martel, and T. Peters (Eds.): MICCAI 2011, Part III, LNCS 6893, pp. 487-495 2011. (C) Springer-Verlag Berlin Heidelberg 2011 
the potential sources of the abnormal electrical events [2]3]. However, there are large variations in the PV drainage patterns [4] and the most common variations (about 20-30\% of the whole population) are extra right PVs and left common PV (where the two left PVs merge into one before joining the chamber). A personalized LA model can help to translate a generic ablation strategy to the specific patient's anatomy, thus making the ablation strategy more effective for this patient. It can also be overlaid onto $2 \mathrm{D}$ real-time fluoroscopic images to provide visual guidance during surgery (Fig. 1).

The previous LA segmentation methods can be roughly categorized as nonmodel based or model based approaches. The non-model based approaches [2 3 . do not assume any prior knowledge of the LA shape and the whole segmentation procedure is purely data driven. For example, John and Rahn [2] proposed to use region growing to extract the highly contrasted blood pool in computed tomography (CT) or magnetic resonance imaging (MRI) data, followed by a split-and-merge procedure to identify the LA. The advantage of these methods is that they can handle structural variations of the PVs. However, such methods cannot provide the underlying anatomical information (e.g., which part of the segmentation is the left inferior PV). In practice, non-model based approaches work well on CT and MRI datasets, but they are not robust on challenging Carm CT. The model based approaches exploit a prior shape of the LA (either in the form of an atlas [5]6] or a mean shape mesh [7]) to guide the segmentation. For example, Manzke et al. [7] built a mean shape of the combined structure of the LA chamber and PVs from a training set. With a prior shape constraint, they could avoid the leakage around weak or missing boundaries, which plagues the non-model based approaches. However, using one mean shape, it is difficult to handle the structural variations, e.g., the left common PV. Furthermore, they did not segment the LA appendage. The PV variations were addressed in [6] using multiple atlases, at the cost of extra computation time.

In this paper, we propose a fully automatic LA segmentation system on Carm CT. Compared to conventional CT or MRI, the advantage of C-arm CT is that overlay of the $3 \mathrm{D}$ patient-specific LA model onto a $2 \mathrm{D}$ fluoroscopic image is straightforward and accurate since both the $3 \mathrm{D}$ and $2 \mathrm{D}$ images are captured on the same device within a short time interval. Normally, a nonelectrocardiography-gated acquisition is performed to reconstruct a C-arm CT volume, therefore, it contains severe cardiac motion artifacts. For a C-arm with a small X-ray detector panel, part of the body may be missing in some $2 \mathrm{D} \mathrm{X}$ ray projections due to the limited field-of-view, resulting in significant artifacts around the margin of a reconstructed volume. In addition, there may be severe streak artifacts caused by various catheters inserted in the heart. These challenges are addressed using a model based approach, which also takes advantage of a machine learning based object pose detector and boundary detector [8].

Instead of using one mean model [7, the PV structural variations are addressed using a part based model, where the whole LA is split into the chamber, appendage, and four major PVs. Each part is a much simpler anatomical structure compared to the holistic one, therefore can be detected and segmented using 

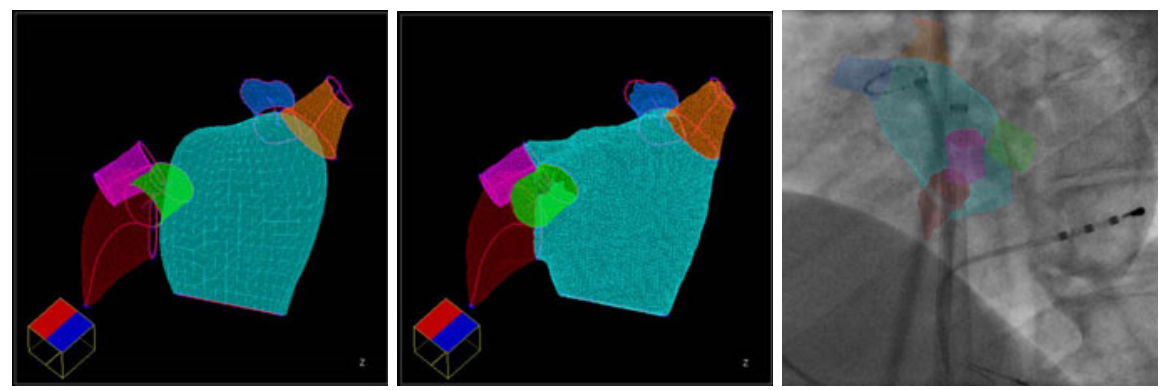

Fig. 1. Part based left atrium (LA) mesh model. Left: Meshes for the separate LA parts. Middle: Final consolidated mesh model. Right: Overlay of the model onto fluoroscopic images to provide visual guidance during surgery. Note: Cyan for the LA chamber, dark red for the appendage, green for the left inferior pulmonary vein (PV), magenta for the left superior PV, orange for the right inferior PV, and blue for the right superior PV.

a model based approach. In order to increase robustness, we detect the most reliable structure (the LA chamber) and use it to constrain the detection of other parts (the appendage and PVs). Experiments show that it is better to treat the LA chamber and appendage as a single object to improve the detection robustness of the appendage. Due to the large variations, the relative position of the PVs to the LA chamber varies quite a lot. A statistical shape model is used to enforce a proper constraint during the estimation of the PV pose parameters (position, orientation, and size). The proposed method is computationally efficient, taking about $1.5 \mathrm{~s}$ to process a volume with $256 \times 256 \times 250$ voxels. Extensive experiments on 469 un-gated C-arm CT datasets demonstrate the robustness of the proposed method.

\section{Multi-part Left Atrium Modeling and Segmentation}

\subsection{Part Based Left Atrium Model}

As shown in the left column of Fig. 1, our part based LA model includes the LA chamber body, appendage, and four major PVs. The shape of the appendage is close to a tilted cone and the PVs have a tubular structure. For AF ablation, physicians only care about a proximal PV trunk, therefore, we only detect a trunk of $20 \mathrm{~mm}$ in length, originating from its ostium. Each LA part is a much simpler anatomical structure compared to the holistic one, therefore can be detected and segmented using a model based approach. We use marginal space learning (MSL) [8] to detect and segment each part. MSL is an efficient method to estimate the position, orientation, and size of the object in a $3 \mathrm{D}$ volume. After automatic object pose estimation, a mean shape is aligned with the pose as an initial mesh. A machine learning based boundary detector [8] is used to guide the boundary evolution. For each LA part, we have an MSL based pose detector and a learning based boundary detector. Interested readers are referred to 8 for 

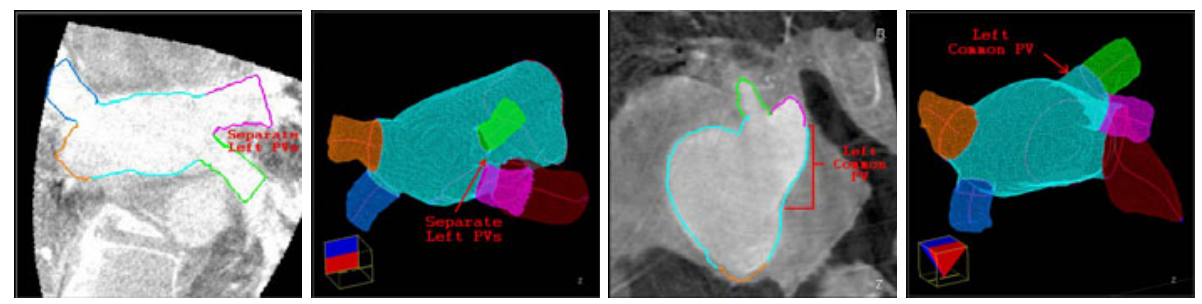

Fig. 2. Pulmonary vein (PV) segmentation results on two datasets. Left: A patient with separate left inferior (green) and superior (magenta) PVs. Right: A patient with the left common PV.

more details of MSL. After detecting/segmenting all parts, we merge them into a consolidated mesh (the middle column of Fig. 11), using the technique presented in Section 2.3.

\subsection{Constrained Detection of LA Parts}

Compared to the holistic approach [7, the part based approach can handle large structural variations. The MSL based detection/segmentation works well for the LA chamber. However, independent detection of other parts is not robust, either due to the low contrast (appendage) or the small object size (PVs). In C-arm CT, the appendage is particularly difficult to detect since the appendage is a pouch without outlet and the blood flow is slow inside the appendage, preventing the complete filling of contrast agent. In many datasets, the appendage is only barely visible. The MSL detector may pick the neighboring left superior PV, which often touches the appendage and has higher contrast. However, the relative position of the appendage to the chamber is quite consistent. The best performance is achieved by treating the appendage and chamber as a consolidated object. One MSL based pose detector is trained to detect the combined object.

Through comparison experiments, we found neither a holistic approach nor independent detection worked for the PVs. In this paper, we propose to enforce a statistical shape constraint [9] in PV detection. The point distribution model (PDM) is often used to enforce the statistical shape constraint among a set of landmarks. The shape variation is decomposed into orthogonal deformation modes through principal component analysis (PCA). A deformed shape is projected into a low dimensional deformation subspace to enforce a statistical shape constraint. For each PV, an MSL detector can estimate nine pose parameters, i.e., three position parameter $\left(T_{x}, T_{y}, T_{z}\right)$, three orientation Euler angles $\left(O_{x}, O_{y}, O_{z}\right)$, and three anisotropic scaling parameters $\left(S_{x}, S_{y}, S_{z}\right)$. Different to the conventional PDM, we also want to enforce the constraint among the estimated orientation and size of PVs. One solution is to stack all PV pose parameters into a big vector to perform PCA. However, the position and orientation parameters are measured in different units. If not weighted properly, the extracted deformation modes may be dominated by one category of transformation. Furthermore, the Euler angles are periodic (with a period of $2 \pi$ ), which 
prevents the application of PCA. Boisvert et al. [10] proposed to build a shape model on a Riemannian manifold on which we have an intrinsic measurement of the orientation distance. However, they still need to heuristically assign a proper weight to the distance in translation w.r.t. the distance in orientation.

In this work, we use a new presentation of the pose parameters to avoid the above problems. The object pose can be fully represented by the object center $T$ together with three scaled orthogonal axes. Alternative to the Euler angles, the object orientation can be represented as a rotation matrix $R=\left(R_{x}, R_{y}, R_{z}\right)$ and each column of $R$ defines an axis. The object pose parameters can be fully represented by a four-point set $\left(T, V_{x}, V_{y}, V_{z}\right)$, where

$$
V_{x}=T+S_{x} R_{x}, \quad V_{y}=T+S_{y} R_{y}, \quad V_{z}=T+S_{z} R_{z} .
$$

The pose of each PV is represented as four points. Besides the constraint among the PVs, we also add the already detected LA chamber center and appendage center to stabilize the detection. In total, we get a set of 18 points.

In our experiments, after detecting the position, orientation, size of the PVs, we project their poses into a subspace with eight dimensions (which explains about $75 \%$ of the total variation) to enforce a statistical shape constraint. After enforcing a statistical shape constraint, the PV center is given by point $\hat{T}$. We can recover the orientation $(\hat{R})$ and scale $(\hat{S})$ by simple inversion of Eq. (1). However, the estimate $\hat{R}$ is generally not a true rotation matrix $\left(\hat{R}^{T} \hat{R} \neq I\right)$. We want to find the nearest rotation matrix $R_{o}$ to minimize the sum of squares of elements in the difference matrix $R_{o}-\hat{R}$, which is equivalent to

$$
R_{o}=\arg \min _{R} \operatorname{Trace}\left((R-\hat{R})^{T}(R-\hat{R})\right),
$$

subject to $R_{o}^{T} R_{o}=I$. Here, Trace(.) is sum of the diagonal elements. The optimal solution [1] is given by

$$
R_{o}=\hat{R}\left(\hat{R}^{T} \hat{R}\right)^{-1 / 2}
$$

\subsection{Mesh Consolidation}

After constrained detection and segmentation, we get six separate meshes (the LA chamber, appendage, and four PVs), as shown in Fig. 1. There may be gaps and/or intersections among different meshes. The physicians prefer a consolidated mesh with different anatomical structures labeled with different colors. We first project the proximal rim of a PV or appendage along the centerline onto the LA chamber to eliminate the gaps among different mesh parts (Fig. 3b). Now, the part meshes are fully connected. However, the mesh intersections may still be present since a piece of the PV mesh may lie inside the chamber. It is complicated to work directly on the meshes. Instead, we convert the meshes to a volume mask (Fig. 35), and generate a new mesh (Fig. 31) from the volume mask using the marching cubes algorithm [12]. Since PV/appendage meshes are connected to the LA chamber in a pure geometric operation, the region around the ostia is not segmented accurately. After converting the meshes to a volume mask, we perform constrained region growing a few layers to improve the segmentation accuracy around the ostia of appendage and PVs. 


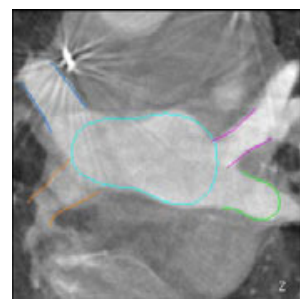

(a)

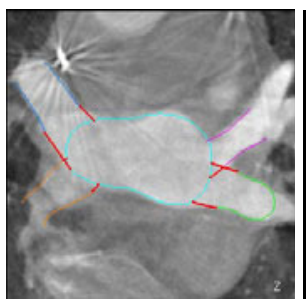

(b)

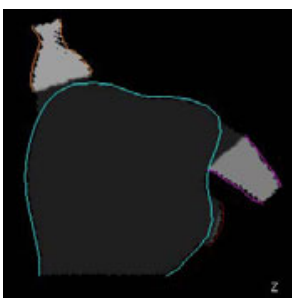

(c)

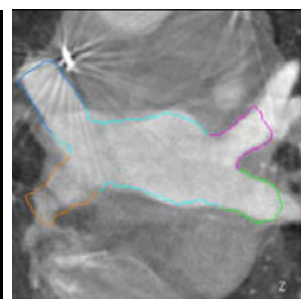

(d)

Fig. 3. Generating a consolidated left atrium (LA) mesh from separate parts. (a) Separate meshes of the pulmonary veins (PV) and LA chamber. (b) After connecting the PV meshes to the LA chamber. The added mesh pieces are shown in red. (c) Volume mask generated from the meshes. (d) Consolidated mesh.

Table 1. Left atrium segmentation errors (based on four-fold cross validation) on C-arm CT datasets with 253 large volumes and 216 small volumes. The symmetric surface-to-surface errors, measured in millimeters $(\mathrm{mm})$, are reported.

\begin{tabular}{|c|c|c|c|c|c|c|c|c|c|c|c|c|}
\hline & \multicolumn{6}{|c|}{ Large Volumes } & \multicolumn{6}{|c|}{ Small Volumes } \\
\hline & \multicolumn{2}{|c|}{ Holistic } & \multicolumn{2}{|c|}{ Independent } & \multicolumn{2}{|c|}{ Proposed } & \multicolumn{2}{|c|}{ Holistic } & \multicolumn{2}{|c|}{ Independent } & \multicolumn{2}{|c|}{ Proposed } \\
\hline & Mean & Median & Mean & Median & Mean & Median & Mean & Median & Mean & Median & Mean & Median \\
\hline LA Chamber & $\overline{1.82}$ & $\overline{1.69}$ & $\overline{1.76}$ & $\overline{1.60}$ & 1.69 & $\overline{1.49}$ & $\overline{2.01}$ & $\overline{1.77}$ & $\overline{1.87}$ & $\overline{1.70}$ & 1.81 & $\overline{1.62}$ \\
\hline Appendage & 3.28 & 2.65 & 3.49 & 2.49 & \begin{tabular}{|l|}
2.89 \\
\end{tabular} & 2.18 & 3.41 & 2.67 & 3.77 & 3.01 & 2.93 & 2.49 \\
\hline Left Inf. PV & 3.08 & 2.49 & 2.01 & 1.59 & \begin{tabular}{|l|}
1.88 \\
\end{tabular} & 1.54 & 4.12 & 2.89 & 2.35 & 1.66 & \begin{tabular}{|l|}
2.01 \\
\end{tabular} & 1.76 \\
\hline Left Sup. PV & 2.77 & 2.21 & 1.82 & 1.42 & \begin{tabular}{|l|}
1.83 \\
\end{tabular} & 1.39 & 3.48 & 2.44 & 2.56 & 1.54 & 1.88 & 1.49 \\
\hline Right Inf. PV & 3.77 & 2.81 & 2.15 & 1.44 & \begin{tabular}{|l|}
1.89 \\
\end{tabular} & 1.43 & 3.93 & 3.09 & 2.34 & 1.66 & \begin{tabular}{|l|}
2.10 \\
\end{tabular} & 1.74 \\
\hline Right Sup. PV & 2.68 & 2.29 & 1.74 & 1.35 & \begin{tabular}{|l|}
1.61 \\
\end{tabular} & 1.40 & 3.08 & 2.50 & 1.72 & 1.35 & 1.62 & 1.31 \\
\hline$\overline{\text { Whole Mesh }}$ & $\overline{2.00}$ & $\overline{1.86}$ & $\overline{1.71}$ & $\overline{1.58}$ & 1.63 & $\overline{\overline{1.51}}$ & $\overline{2.17}$ & $\overline{1.98}$ & $\overline{\overline{1.79}}$ & $\overline{1.63}$ & $\overline{1.71}$ & $\overline{1.55}$ \\
\hline
\end{tabular}

\section{$3 \quad$ Experiments}

We collected 469 C-arm CT datasets, scanned by Siemens Axiom Artis zee Carm systems at 17 clinical sites from Europe and the USA. Among them, 253 datasets were scanned with large X-ray detector panels $\left(30 \times 40 \mathrm{~cm}^{2}\right)$ with an isotropic volume resolution of $0.30 \mathrm{~mm}^{3}$. The other 216 datasets were scanned with small X-ray detectors $\left(20 \times 20 \mathrm{~cm}^{2}\right)$ with a volume resolution of $0.18 \mathrm{~mm}^{3}$. Due to the limited field-of-view of a small X-ray detector, the reconstructed volumes may contain significant artifacts, especially around the volume margin. Due to the heterogeneity of the datasets, we train two separate systems for the large and small volumes, respectively.

A four-fold cross validation is performed to evaluate our algorithm and the segmentation accuracy is measured using the symmetric surface-to-surface distance. Table 1 shows segmentation errors of the consolidated mesh using various approaches. For a mesh part, we measure the distance to the corresponding part in the ground truth, therefore both the segmentation and mesh part labeling errors are penalized. At the bottom row of Table 1, we also list the whole mesh segmentation error without considering the mesh part labeling. If we treat the whole LA mesh as a holistic object, the segmentation errors are large, especially 

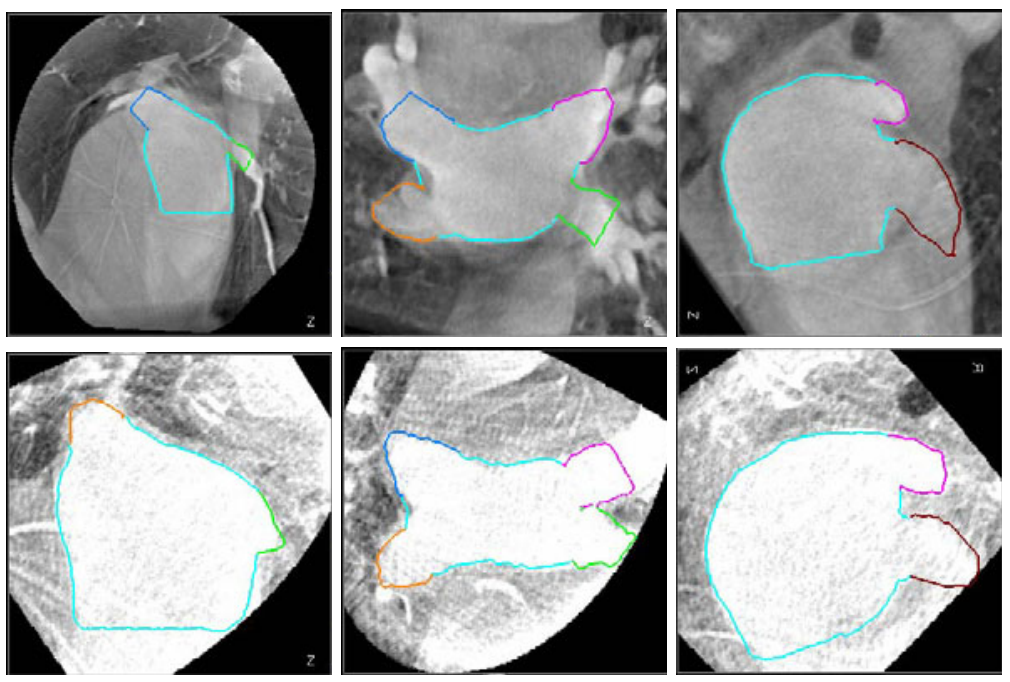

Fig. 4. The left atrium segmentation results on a large volume (top row) and a small volume (bottom row) with different colors for different mesh parts. Three different views are shown for each volume.

for the PVs due to the structural variations. Independent detection of the parts can significantly improve the PV segmentation accuracy. However, the LA appendage segmentation is deteriorated. Using the proposed method, we improve the segmentation for all LA parts. Compared to the independent detection, on average, we achieve about $10 \%$ reduction in mean error for PVs (up to $26.6 \%$ reduction for the left superior $\mathrm{PV}$ on small volumes). Inferior PVs have larger errors than superior PVs since they are more likely to be cut by the volume borders.

We cannot directly compare our segmentation accuracy with those reported in the literature due to the difference in imaging modalities, datasets, and LA models. There is no quantitative evaluation available in 23. To the best of our knowledge, there is only one previous work [7] on the LA segmentation on C-arm $\mathrm{CT}$, which was validated on large volumes only and did not segment the more challenging LA appendage. After excluding the LA appendage, we achieve an overall segmentation error of $1.50 \mathrm{~mm}$ for large volumes and $1.63 \mathrm{~mm}$ for small volumes. In [7], a mean segmentation error of $1.3 \mathrm{~mm}$ is reported on 33 datasets (which is significantly smaller than ours). Their segmentation is initialized with a holistic mean shape. It is not clear if patients with the left common PV are included in their limited datasets, since a holistic approach has difficulty to handle such structural variations. We also notice that the mesh triangles near the distal bounds of the truncated PVs (9.4\% of all triangles) are excluded from the error measurement, however, we include all mesh triangles in evaluation.

The proposed method is computationally efficient, taking about $1.5 \mathrm{~s}$ (on a computer with quad-core $2.33 \mathrm{GHz}$ CPUs and $3 \mathrm{~GB}$ memory) to process a 
volume with $256 \times 256 \times 250$ voxels. It compares favorably with the previous methods, e.g., $5 \mathrm{~s}$ in [5], 5-45 $\mathrm{s}$ in [2, $30 \mathrm{~s}$ in [7], and more than two hours in [6].

\section{Conclusion}

We proposed a part based model for the automatic segmentation of the LA (including chamber, appendage, and four major PVs) in C-arm CT volumes, which can handle structural variations elegantly. Extensive experiments on 469 ungated C-arm CT datasets demonstrated the robustness and efficiency of the proposed method. As an extension, we are currently working on the automatic segmentation of the extra right PVs if they are present. Our system is re-trainable, therefore can be extended to different medical imaging modalities, e.g., MRI and conventional CT.

\section{References}

1. Lloyd-Jones, D., Adams, R., Carnethon, M., et al.: Heart disease and stroke statistics - 2009 update. Circulation 119(3), 21-181 (2009)

2. John, M., Rahn, N.: Automatic left atrium segmentation by cutting the blood pool at narrowings. In: Duncan, J.S., Gerig, G. (eds.) MICCAI 2005. LNCS, vol. 3750, pp. 798-805. Springer, Heidelberg (2005)

3. Karim, R., Mohiaddin, R., Rueckert, D.: Left atrium segmentation for atrial fibrillation ablation. In: Proc. of SPIE Medical Imaging (2008)

4. Marom, E.M., Herndon, J.E., Kim, Y.K., McAdams, H.P.: Variations in pulmonary venous drainage to the left atrium: Implications for radiofrequency ablation. Radiology 230, 824-829 (2004)

5. Karim, R., Juli, C., Lawes, L.M., Kanangaratnam, P., Davies, D.W., Peters, N.S., Rueckert, D.: Automatic segmentation of left atrial geometry from contrastenhanced magnetic resonance images using a probabilistic atlas. In: Camara, O., Pop, M., Rhode, K., Sermesant, M., Smith, N., Young, A. (eds.) STACOM 2010. LNCS, vol. 6364, pp. 134-143. Springer, Heidelberg (2010)

6. Depa, M., Sabuncu, M.R., Holmvang, G., Nezafat, R., Schmidt, E.J., Golland, P.: Robust atlas-based segmentation of highly variable anatomy: Left atrium segmentation. In: Camara, O., Pop, M., Rhode, K., Sermesant, M., Smith, N., Young, A. (eds.) STACOM 2010. LNCS, vol. 6364, pp. 85-94. Springer, Heidelberg (2010)

7. Manzke, R., Meyer, C., Ecabert, O., Peters, J., Noordhoek, N.J., Thiagalingam, A., Reddy, V.Y., Chan, R.C., Weese, J.: Automatic segmentation of rotational Xray images for anatomic intra-procedural surface generation in atrial fibrillation ablation procedures. IEEE Trans. Medical Imaging 29(2), 260-272 (2010)

8. Zheng, Y., Barbu, A., Georgescu, B., Scheuering, M., Comaniciu, D.: Four-chamber heart modeling and automatic segmentation for 3D cardiac CT volumes using marginal space learning and steerable features. IEEE Trans. Medical Imaging 27(11), 1668-1681 (2008)

9. Cootes, T.F., Taylor, C.J., Cooper, D.H., Graham, J.: Active shape models - their training and application. Computer Vision and Image Understanding 61(1), 38-59 (1995) 
10. Boisvert, J., Pennec, X., Labelle, H., Cheriet, F., Ayache, N.: Principal spine shape deformation modes using Riemannian geometry and articulated models. In: Perales, F.J., Fisher, R.B. (eds.) AMDO 2006. LNCS, vol. 4069, pp. 346-355. Springer, Heidelberg (2006)

11. Horn, B.K.P.: Closed form solution of absolute orientation using unit quaternions. Journal of the Optical Society A 4(4), 629-642 (1987)

12. Lorensen, W.E., Cline, H.E.: Marching cubes: A high resolution 3D surface construction algorithm. Computer Graphics 21(4), 163-169 (1987) 